\title{
POLIMORFISMOS DEL GEN ARSÉNICO 3 METILTRANSFERASA (AS3MT) Y LA EFICIENCIA URINARIA DEL METABOLISMO DEL ARSÉNICO EN UNA POBLACIÓN DEL NORTE DE MÉXICO
}

\begin{abstract}
Francisco Javier García-Alvarado ${ }^{1, a}$, Honorio Neri-Meléndez ${ }^{2, b}$, Luz Pérez Armendáriz ${ }^{1, a}$, Mario Rivera Guillen ${ }^{1, a}$
RESUMEN

El objetivo del estudio fue evaluar la relación de los polimorfismos rs11191439 y rs3740393 del gen Arsénico 3 Metiltransferasa (As3MT) con los perfiles de excreción urinaria de arsénico, en una población del norte de México. Se realizó un estudio transversal con 102 participantes de 14 a 75 años en la Comarca Lagunera, México. Se determinó la especiación de los metabolitos urinarios de arsénico y extracción de ADN, el análisis y amplificación de los polimorfismos se realizó por PCR tiempo real. Se realizó un análisis descriptivo y se evaluó las diferencias en las concentraciones de arsénico urinario por sexo y genotipo. La mediana de concentración de arsénico en el agua fue de $82 \mu \mathrm{g} / \mathrm{L}$, los niveles de excreción urinaria del ácido dimetilarsínico (DMA) fueron más altos en mujeres que en los hombres. Los portadores de la variante genética CC del gen As3MT (rs3740393) presentaron concentraciones urinarias superiores de ácido monometilarsónico $(p=0,01)$ y DMA $(p=0,05)$.
\end{abstract}

Palabras clave: Arsénico; Polimorfismo genético; Orina; Genotipo (fuente: DeCS BIREME)

\section{POLYMORPHISMS OF THE ARSENITE METHYLTRANSFERASE (AS3MT) GENE AND URINARY EFFICIENCY OF ARSENIC METABOLISM IN A POPULATION IN NORTHERN MEXICO}

\begin{abstract}
The aim of this study was to assess the relationship of the rs11191439 and rs3740393 polymorphisms of the Arsenite Methyltransferase (As3MT) gene with the arsenic urinary excretion profiles in a population in northern Mexico. A crosssectional study was conducted with 102 participants between the ages of 14 and 75 in Comarca Lagunera, Mexico. Speciation of the arsenic urinary metabolites was determined, and DNA was extracted; analysis and amplification of the polymorphisms was made by PCR in real time. A descriptive analysis was carried out and the differences in the urinary arsenic concentrations were assessed according to sex and genotype. The median concentration of arsenic in water was $82 \mathrm{\mu g} / \mathrm{L}$; the levels of urinary excretion of dimethylarsinic acid (DMA) were higher in women than in the men. The carriers of the CC genetic variant of the As3MT (rs3740393) gene showed higher urinary concentrations of methylarsinic acid $(p=0.01)$ and DMA $(p=0.05)$.
\end{abstract}

Keywords: Arsenic; Genetic polymorphism; Urine; Genotype (source: MeSH NLM)

\section{INTRODUCCIÓN}

El hidroarsenicismo crónico es el consumo de arsénico en agua por largos periodos de tiempo; esto se considera un problema de salud pública a nivel mundial (1). En México, al menos cuatro millones de personas beben, en forma permanente, agua con concentraciones altas de este tóxico (2). La ingesta de arsénico inorgánico (Asi) en agua de consumo humano se ha asociado con lesiones de la piel (premalignas y malignas) y cáncer de tipo renal, pulmonar y de vejiga ${ }^{(3,4)}$. Además, la exposición prolongada a este tóxico está asociado al desarrollo de enfermedades crónicas degenerativas como hipertensión, diabetes mellitus tipo 2, alteraciones vasculares, renales y neurológicas ${ }^{(5,6)}$. Este tipo de efectos nocivos también se han descrito en México, donde existe elevadas concentraciones de arsénico en el agua de consumo humano ${ }^{(7)}$.

EI Asi se absorbe eficientemente en el tracto gastrointestinal y se metaboliza en el cuerpo por una serie de reacciones de

\footnotetext{
Centro de Investigación Biomédica, Universidad Autónoma de Coahuila. Coahuila, México.

Facultad de Medicina Torreón, Universidad Autónoma de Coahuila. Coahuila, México

Doctor en Ciencias Biomédicas; ${ }^{b}$ maestro en Investigación en Salud

Recibido: 25/09/2017 Aprobado: 21/02/2018 En línea: 23/03/2018
}

Citar como: García-Alvarado FJ, Neri-Meléndez H, Pérez Armendáriz L, Rivera Guillen M. Polimorfismos del gen Arsénico 3 Metiltransferasa (As3MT) y la eficiencia urinaria del metabolismo del arsénico en una población del norte de México. Rev Peru Med Exp Salud Publica. 2018;35(1):72-6. doi: 10.17843/rpmesp.2018.351.3565. 
reducción y metilación; como resultado del metabolismo del Asi se obtiene el ácido monometilarsónico (MMA) y el ácido dimetilarsínico (DMA), ambos son excretados en la orina ${ }^{(8)}$. Existe una gran variabilidad en la capacidad para metilar el arsénico entre individuos, la metilación eficiente del Asi se asocia con una alta tasa de excreción de DMA en la orina. El metabolito de arsénico mas tóxico es MMA, los niveles bajos de MMA en comparación con DMA en la orina son beneficiosos para reducir su toxicidad ${ }^{(9)}$. Esta variabilidad se ha asociado a diversos factores, tales como el sexo, la edad, el nivel de exposición, la presencia de polimorfismo en genes codificantes de enzimas relacionadas con el metabolismo del arsénico.

Arsénico 3 Metiltransferasa (AS3MT) es una de las enzimas involucradas en la transferencia de un grupo metilo de s-adenosil metionina a arsénico trivalente, que juega un papel crítico en la desintoxicación del arsénico. El efecto de los polimorfismos genéticos de AS3MT está relacionado directamente con el aumento o disminución de la excreción el arsénico, esto puede conducir a diferencias en el metabolismo y la toxicidad asociada con la exposición de este metal ${ }^{(10,11)}$. Recientes estudios en poblaciones indígenas en los Andes demuestran una eficiente excreción del arsénico, con una baja excreción urinaria de MMA y alta excreción de DMA ${ }^{(12)}$.

Por lo anterior, se planteó como objetivo evaluar la relación de los polimorfismos rs11191439 y rs3740393 del gen As3MT con los perfiles de excreción urinarios de arsénico en una población del norte de México.

\section{EL ESTUDIO}

Estudio transversal realizado en comunidades rurales de la Comarca Lagunera ubicada en la región norte de México con altas concentraciones de arsénico en el agua de bebida que exceden los límites permisibles de $(25 \mu \mathrm{g} / \mathrm{L})$ establecido en la NOM-127-SSA1-1994 (13).

Para el cálculo de la muestra se tuvo como referencia la prevalencia de hidroarsenisismo en la región lagunera del norte de México, teniendo en cuenta una precisión del $5 \%$ y confiabilidad del $95 \%$. Se realizó un muestreo multietápico con la selección de los individuos por muestreo aleatorio simple. Las comunidades seleccionadas se estratificaron en exposición baja ( $\leq 25 \mu \mathrm{g} / \mathrm{L})$ y exposición alta $(>25 \mu \mathrm{g} / \mathrm{L})$.

Se incluyeron adultos de 14 a 75 años que habitan en comunidades rurales de la Comarca Lagunera, cuyas muestras domiciliarias de agua presenten altas concentraciones de arsénico. Se excluyeron a individuos con información incompleta y muestras degradadas. Los individuos que aceptaron participar se les aplicó un cuestionario estandarizado y validado por la comisión

\section{MENSAJES CLAVE}

Motivación para realizar el estudio. La contaminación del agua provocada por el arsénico es un grave problema de salud pública a nivel mundial.

Principales hallazgos. El presente estudio encontró altas concentraciones de arsénico en el agua, los niveles de excreción urinaria del ácido dimetilarsínico (DMA) fueron más altos en mujeres que en los hombres. Los habitantes de la muestra seleccionada excretan de forma eficiente el metabolito DMA, lo que podrían estar influenciado por las variantes genéticas del gen AS3MT.

Implicancias. Estos resultados permitirán a profesionales de la salud evaluar el impacto de la ingesta de arsénico en el agua, con la finalidad de elaborar estrategias de intervención en salud ambiental.

federal para la protección contra riesgos sanitarios de la secretaria de salud del estado de Coahuila.

La recolección de las muestras de agua se realizó en las tomas domiciliarias de los participantes, en frascos de un litro almacenados a un $\mathrm{pH}$ menor de 2 y conservadas en congelación a $-18{ }^{\circ} \mathrm{C}$. La concentración de arsénico total se determinó utilizando un equipo de espectrofotometría de absorción atómica (Perkin Elmer AANALYST 800). Las muestras de sangre se obtuvieron por punción venosa y se recolectaron en tubos $B D$ vacutainer con anticoagulante EDTA, el aislamiento del ADN se realizó siguiendo lo descrito por el método de extracción de salting-out ${ }^{(14)}$. Las muestras de orina se recolectaron de la primera micción (por la mañana) de los participantes, en un recipiente de plástico y se almacenaron a $-80^{\circ} \mathrm{C}$.

La amplificación de los genotipos se determinó por PCR en tiempo real, utilizando el software Applied Biosystems 7300 Real Time PCR System (Thermo Fischer, Scientific, Waltham, MA, USA). En un tubo de PCR de tapa con calidad óptica, se añadieron $10 \mu \mathrm{l}$ de Master Mix TaqMan Genotyping y $0,5 \mu \mathrm{L}$ que incluía los dos cebadores y una sonda fluorescente específica para cada uno de los polimorfismos. Se añadieron $3 \mu \mathrm{L}$ a la solución que contenía la sonda de $\operatorname{ADN}(10 \mathrm{ng}$ ) y se llevaron a un volumen final de $20 \mu \mathrm{L}$ añadiendo agua deionizada y bidestilada (reactivos de Applied Biosystems, CA, EE. UU.). La discriminación alélica se desarrolló utilizando el sistema de software del fabricante (Applied Biosystem, CA, EUA).

La determinación de arsénico en orina se realizó en $1 \mathrm{ml}$ de orina, la cual se digirió con $\mathrm{NaOH} 2 \mathrm{M}$ a $95^{\circ} \mathrm{C}$ durante 3 horas. Posteriormente se centrifugó por $30 \mathrm{~min}$. Las muestras tratadas se diluyeron con $10 \mathrm{ml}$ de $\mathrm{HNO}$ (Ácido nítrico) y $\mathrm{HClO} 4$ (Ácido perclórico) en una relación 1:6. La determinación de Asi, MMA y DMA se realizó con un espectrofotómetro de absorción atómica AAnalyst 800 Atomic Absorption Spectrometer (Perkin Elmer, Waltham, Massachusetts, EUA) con un sistema de pretratamiento de la especiación de arsénico (ASA-2SP, Shimadzu; ShenZhen, China), según el método descrito ${ }^{(15)}$. Las concentraciones de 
arsénico urinario se ajustaron por los niveles de creatinina utilizando el método colorimétrico de Jaffe, el análisis se llevó a cabo a $520 \mathrm{~nm}$ en un espectrómetro Lambda EZ210 (Perkin Elmer; Waltham, Massachusetts, EUA) con el fin de ajustar los niveles de excreción urinario de los participantes del estudio.

Asimismo, se determinó la existencia de diferencias entre los genotipos según el modelo genético recesivo $(A A+A a)$ (aa), el cual combina el genotipo homocigoto silvestre (AA) más el genotipo heterocigoto $(\mathrm{Aa})$, luego este se compara con el genotipo homocigoto mutado (aa).

\section{ANÁLISIS ESTADÍSTICO}

Como medida de tendencia central y de dispersión se utilizó a la media y la desviación estándar en variables con distribución normal. Se utilizó la mediana y el rango intercuartilico en las variables con distribución asimétrica. Para el cálculo de los metabolitos de arsénico se realizó una conversión a su logaritmo natural para aproximarse a una distribución normal. Las variables categóricas fueron presentadas como frecuencias y porcentajes. Se procedió al análisis descriptivo de las variables, además se realizó la prueba de chi cuadrado para evaluar si los genotipos se encontraban en equilibrio de Hardy-Weinberg. Para determinar la diferencia entre las medianas de los metabolitos de arsénico según sexo y genotipo se utilizó la prueba de $U$ de Mann Whitney. Para el análisis de los datos se utilizó el programa estadístico STATA 14 (Stata Corp., College Station, TX). Se aceptó un valor de $p$ menor o igual a 0,05 como estadísticamente significativo.

\section{CONSIDERACIONES ÉTICAS}

El protocolo del estudio fue aprobado por el comité de ética de la Facultad de Medicina de Torreón, Universidad de Coahuila, México.

\section{RESULTADOS}

Se incluyeron 102 individuos, con un promedio de edad de 35,9 años, con rangos desde los 14 a 75 años. El 55,9 \% fueron mujeres y el $44,1 \%$ hombres. Las comunidades seleccionadas tienen un promedio de ingesta de agua de 2 L/día, con una concentración promedio de arsénico en el agua de $82,3 \pm 1,2 \mu \mathrm{g} / \mathrm{L}$. Las distribuciones del genotipo según los polimorfismos se encontraban en equilibrio de Hardy-Weinberg $(p>0,05)$ (Tabla 1).

Las concentraciones de arsénico urinario, ajustados por creatinina, se compararon por sexo. Las mujeres presentaron un mediana de excreción urinaria de DMA de $67,4 \%$ mientras que los hombres tuvieron una excreción de $57,8 \%$, siendo esta diferencia significativa $(p=0,05)$ (Tabla 2).

Las concentraciones de arsénico en orina se compararon por genotipo de los polimorfismos rs11191439 y rs3740393
Tabla 1. Características generales de los participantes del estudio

\begin{tabular}{|c|c|}
\hline Características & $n=102$ \\
\hline Edad (años), media $\pm \mathrm{DE}$ & $35,9 \pm 2,3$ \\
\hline \multicolumn{2}{|l|}{ Sexo, n (\%) } \\
\hline Mujer & $57(55,9)$ \\
\hline Hombre & $45(44,1)$ \\
\hline \multicolumn{2}{|l|}{ Fuma, n (\%) } \\
\hline $\mathrm{Si}$ & $40(39,2)$ \\
\hline No & $62(60,8)$ \\
\hline \multicolumn{2}{|l|}{ Trabaja, n (\%) } \\
\hline $\mathrm{Si}$ & $90(88,2)$ \\
\hline No & $12(11,8)$ \\
\hline As total $(\mu \mathrm{g} / \mathrm{L})$ media $\pm \mathrm{DE}$ & $53 \pm 1,5$ \\
\hline \multicolumn{2}{|c|}{ Metabolitos de arsénico, media $\pm \mathrm{DE}$} \\
\hline$\%$ DMA & $34,1 \pm 1,2$ \\
\hline$\%$ MMA & $5,9 \pm 1,3$ \\
\hline$\%$ Asi & $13,6 \pm 0,4$ \\
\hline \multicolumn{2}{|c|}{ Estatus de Exposición de arsénico, n (\%) } \\
\hline Alto $(>25 \mu \mathrm{g} / \mathrm{L})$ & $51(50,0)$ \\
\hline Bajo $(<25 \mu \mathrm{g} / \mathrm{L})$ & $51(50,0)$ \\
\hline \multicolumn{2}{|l|}{ Genotipo, n (\%) } \\
\hline \multicolumn{2}{|l|}{ AS3MT, rs11191439 } \\
\hline $\mathrm{TT}$ & $73(71,5)$ \\
\hline TC & $16(15,6)$ \\
\hline $\mathrm{CC}$ & $13(12,9)$ \\
\hline $\mathrm{H}-\mathrm{W}$ & $0,095^{*}$ \\
\hline \multicolumn{2}{|l|}{ AS3MT, rs3740393 } \\
\hline $\mathrm{CC}$ & $57(55,8)$ \\
\hline CG & $38(37,3)$ \\
\hline GG & $7(6,9)$ \\
\hline $\mathrm{H}-\mathrm{W}$ & $0,073^{*}$ \\
\hline
\end{tabular}

DE: Desviación estándar, \%: porcentaje

As total: Asi+ MMA+ DMA, Asi: arsénico inorgánico, MMA: ácido monometilarsónico, DMA: ácido dimetilarsínico

Estatus de exposición alto (concentración mayor de $25 \mu \mathrm{g} / \mathrm{L}$ de As en agua) Estatus de exposición bajo (concentración menor de $25 \mu \mathrm{g} / \mathrm{L}$ de As de agua) Genotipo AA: homocigoto silvestre, Aa: heterocigoto, aa: homocigoto mutado

H-W: equilibrio de Hardy-Weinberg

*Valor de $p$, prueba de chi cuadrado

del gen AS3MT. En el modelo recesivo, el polimorfismo del gen AS3MT (rs3740393) en el genotipo homocigoto mutado presento concentraciones urinarias superiores de MMA $(p=0,01)$ y DMA $(p=0,05)$ con una diferencia estadísticamente significativa en comparación con el genotipo homocigoto de tipo silvestre y heterocigotos que tenían concentraciones más bajas de Asi y MMA en orina. El polimorfismo en el gen AS3MT (rs11191439) tuvieron una diferencia significativa $(p=0,03)$ en las concentraciones urinarias de MMA (Tabla 3). 
Tabla 2. Excreción de los metabolitos de arsénico en orina según sexo

\begin{tabular}{lrrr}
\hline Metabolitos, mediana (RIC) & Hombres $(\mathbf{n}=\mathbf{4 5})$ & Mujeres $(\mathbf{n}=\mathbf{5 7})$ & \multicolumn{1}{c}{ Valor de $\boldsymbol{p}^{*}$} \\
\hline \% Asi & $21,7(15,9-29,2)$ & $23,9(16,5-34,5)$ & 0,38 \\
\% MMA & $10,2(7,5-13,1)$ & $10,8(8,2-13,5)$ & 0,12 \\
$\%$ DMA & $57,8(42,1-60,9)$ & $67,4(52,5-72,5)$ & 0,05 \\
\hline
\end{tabular}

RIC: rango intercuartilico (percentil 25 al percentil 75 )

Asi: arsénico inorgánico, MMA: ácido monometilarsónico, DMA: ácido dimetilarsínico

*Prueba de U de Mann-Whitney

\section{DISCUSIÓN}

Estudios en poblaciones expuestas crónicamente al arsénico del agua potable han demostrado que existe una gran variabilidad interindividual en la distribución de los metabolitos de arsénico urinario ${ }^{(16)}$. La población de estudio presentó una mediana de concentraciones de arsénico en el agua de $82,3 \mu \mathrm{g} / \mathrm{L}$, excediendo los límites permisibles en la norma mexicana.

Entre los principales factores que intervienen en el metabolismo del arsénico se encuentra el sexo, la mayoría de los estudios sugieren que las mujeres tienen una capacidad de metilación y excreción de arsénico más eficiente que los hombres; posiblemente asociado a factores hormonales ${ }^{(17,18)}$. Estudios recientes han demostrado que algunas variantes genéticas están asociadas con la eficiente metabolización de arsénico, lo que sugiere que las poblaciones expuestas durante largos períodos de tiempo pueden poseer algún tipo de protección contra la toxicidad de este elemento ${ }^{(19)}$.

Tabla 3. Concentraciones de arsénico en orina por genotipo utilizando el modelo recesivo

\begin{tabular}{|c|c|c|c|}
\hline \multirow{3}{*}{$\begin{array}{c}\text { Metabolito } \\
\text { mediana } \\
(\mathrm{RIC})\end{array}$} & \multirow{2}{*}{\multicolumn{2}{|c|}{$\begin{array}{c}\text { Genotipos } \\
\text { AS3MT (rrs3740393) }\end{array}$}} & \multirow{3}{*}{$\begin{array}{l}\text { Valor } \\
\text { de } p^{*}\end{array}$} \\
\hline & & & \\
\hline & $\mathrm{TC}+\mathrm{TT}$ & $\mathrm{CC}$ & \\
\hline$\%$ Asi & $22,4(15,9-33,2)$ & $21,1(15,8-31,7)$ & 0,49 \\
\hline$\%$ MMA & $10,8(7,8-13,5)$ & $12,4(6,3-15,1)$ & 0,01 \\
\hline$\%$ DMA & $66,1(55,4-72,7)$ & $77,5(41,9-80,1)$ & 0,05 \\
\hline \multicolumn{4}{|c|}{ AS3MT (rs11191439) } \\
\hline & CG+ GG & CC & \\
\hline$\%$ Asi & $19,8(14,5-29,3)$ & $20,4(15,6-31,9)$ & 0,45 \\
\hline$\%$ MMA & $11,1(10,1-12,3)$ & $11,8(9,2-13,9)$ & 0,03 \\
\hline$\%$ DMA & $58,6(53,5-63,7)$ & $68,2(65,5-71)$ & 0,79 \\
\hline
\end{tabular}

RIC: rango intercuartilico (percentil 25 al percentil 75)

Asi: arsénico inorgánico, MMA: ácido monometilarsónico, DMA: ácido dimetilarsínico

Modelo recesivo ( $\mathrm{AA}+\mathrm{Aa})(\mathrm{aa})$

Genotipos AA: homocigoto silvestre, Aa: heterocigoto aa: homocigoto mutado

* Prueba de U de Mann Whitney
Un estudio reciente demostró que existe una asociación entre el daño genotípico delADN en los leucocitos de sangre periférica y la frecuencia del alelo variante rs11191439 (Met287Thr) del gen As3MT en niños que viven en la región de Coahuila, México; también se asociaron con una mayor proporción de MMA en los niños ${ }^{(20)}$. Cabe señalar que en nuestro estudio no se evaluó el daño genotóxico y no se incluyeron niños, pero se encontraron altos niveles de MMA en los portadores del polimorfismo rs11191439 (Met287Thr) en personas adultas.

Las poblaciones indígenas en los Andes demuestran un metabolismo eficiente del arsénico, con baja excreción urinaria de MMA y alta excreción de DMA (11). Nuestros hallazgos demuestran que la población de estudio con la variante genética CC del gen AS3MT (rs3740393) tiene una eficiente excreción del metabolismo del arsénico, con una mayor excreción de DMA.

Como limitaciones del estudio podemos mencionar que solo se realizó una medición de las excreciones urinarias de arsénico, por lo que no se pudo evaluar el efecto de la temporalidad. Se recomiendan la realización de más trabajos de seguimiento que permitan evaluar el efecto de la ingesta crónica de arsénico en poblaciones expuestas.

En conclusión, los habitantes con la variante genética de AS3MT (rs3740393) de la muestra seleccionada, excretan de forma eficiente el metabolito urinario DMA y así reducen sus efectos nocivos en la salud. Los polimorfismos de un solo nucleótido (SNP) del gen AS3MT tienen un papel muy importante en el metabolismo y la excreción de arsénico.

Contribuciones de autoría: FJGA, RGM, HNM y AL, participaron en la concepción del estudio, recolección de resultados, análisis e interpretación de datos, redacción del manuscrito, revisión crítica del artículo y la aprobación de la versión final a publicar. RGM y FJGA contribuyeron en recolección de datos y diseño del estudio, $\mathrm{HNM}$ y $\mathrm{AL}$ contribuyeron en el procesamiento de las muestras, HNM y FJGA contribuyeron en la creación de la base de datos y el análisis estadístico para genética de poblaciones.

Fuentes de financiamiento: Este trabajo ha recibido el apoyo financiero de la Facultad de Medicina de la Universidad Autónoma de Coahuila, Unidad Torreón.

Conflictos de interés: Los autores declaran no tener conflictos de interés en la publicación de este artículo. 


\section{REFERENCIAS BIBLIOGRÁFICAS}

1. van Halem D, Bakker SA, Amy GL, van Dijk J. Arsenic in drinking water: a worldwide water quality concern for water supply companies. Drink Water Eng Sci. 2009;2(1):29-34.

2. Loffredo CA, Aposhian HV, Cebrian ME, Yamauchi H, Silbergeld EK. Variability in human metabolism of arsenic. Environ Res. 2003;92(2):85-91.

3. National Research Council. Arsenic in Drinking Water, 2001 Update. WashingtonD.C:TheNationalAcademies Press; 2001. doi: 10.17226/10194.

4. World Health Organization. Some drinking-water disinfectants and contaminants, including arsenic /IARC Working Group on the Evaluation of Carcinogenic Risks to Humans [Internet]. Lyon: IARCPress; 2004. Disponible en: http://monographs.iarc.fr/ENG/Monographs/vol84/ mono84.pdf

5. James K, Marshall J, Hokanson J, Meliker J, Zerbe G, Byers T. A case-cohort study examining lifetime exposure to inorganic arsenic in drinking water and diabetes mellitus. Environ Res. 2013;123:33-8. doi: 10.1016/j.envres.2013.02.005.

6. Chiou J, Wang S, Chen C, Deng C, Lin W, Tai T. Arsenic ingestion and increased microvascular disease risk: observations from the south-western arseniasis-endemic area in Taiwan. Int J Epidemiol. dyi108.

7. Cebrian M, Albores A, Aguilar M, Blakely E. Chronic Arsenic Poisoning in the North of Mexico. Hum Toxicol. 1983;2(1):121-33.

8. Vahter M. Mechanisms of arsenic biotransformation. Toxicology. 2002;181182:211-7.
9. Smith A, Steinmaus C. Health Effects of Arsenic and Chromium in Drinking Water: Recent Human Findings. Annu Rev Public Health. 2009;30:107-22. doi: 10.1146/annurev.publhealth.031308.100143.

10. Hughes M. Biomarkers of Exposure: A Case Study with Inorganic Arsenic. Environ Health Perspect. 2006;114(11):1790-6.

11. Wood T, Salavagionne O, Mukherjee B, Wang L, Klumpp A, Thomae B, et al. Human Arsenic Methyltransferase (AS3MT) Pharmacogenetics: gene resequencing and functional genomics studies. J Biol Chem. 2006;281(11):7364-73.

12. Vahter M, Concha G, Nermell B, Nilsson R, Dulout F, Natarajan A. A unique metabolism of inorganic arsenic in native Andean women. Eur J Pharmacol. 1995;293(4):455-62.

13. Salud ambiental, agua para uso y consumo humano-limites permisibles de calidad y tratamientos a que debe someterse el agua para su potabilización. Norma oficial mexicana NOM-127-SSA1-1994. Secretaría de Salud de México (22-112000). Disponible en: http://www.salud. gob.mx/unidades/cdi/nom/127ssa14 html

14. Miller S, Dykes D, Polesky H. A simple salting out procedure for extracting DNA from human nucleated cells. Nucleic Acids Res. 1988;16(3):1215.

15. Del Razo L, Styblo M, Cullen W, Thomas D. Determination of Trivalent Methylated Arsenicals in Biological Matrices. Toxicol Appl Pharmacol. 2001;174(3):282-93. doi: 10.1006/taap.2001.9226
16. Lindberg A, Ekström E, Nermell B, Rahman M, Lönnerdal B, Persson L et al. Gender and age differences in the metabolism of inorganic arsenic in a highly exposed population in Bangladesh. Environ Res. 2008;106(1):110-20. doi: 10.1016/j.envres.2007.08.011.

17. Vahter ME, Li L, Nermell B, Rahman A, El Arifeen S, Rahman M, et al. Arsenic exposure in pregnancy: a populationbased study in Matlab, Bangladesh. J Health Popul Nutr. 2006;24(2):236-45.

18. Fischer LM, daCosta KA, Kwock L, Stewart PW, Lu TS, Stabler SP, et al. Sex and menopausal status influence human dietary requirements for the nutrient choline. Am J Clin Nutr. 2007;85(5):1275-85.

19. Eichstaedt C, Antao T, Cardona A, Pagani L, Kivisild T, Mormina M. Positive selection of AS3MT to arsenic water in Andean populations. Mutat Res. 2015;780:97-102. doi: 10.1016/j. mrfmmm.2015.07.007.

20. Sampayo-Reyes A, Hernández A, El-Yamani N, López-Campos C, Mayet-Machado E, Rincón-Castañeda C, et al. Arsenic Induces DNA Damage in Environmentally Exposed Mexican Children and Adults. Influence of GSTO1 and AS3MT Polymorphisms. Toxicol Sci. 2010;117(1):63-71. doi: $10.1093 /$ toxsci/kfq173.

Correspondencia: Mario Rivera Guillen Dirección: Facultad de Medicina, Universidad Autónoma de Coabuila, Unidad Torreón, calle Gregorio A García 198. Coabuila, México Teléfono: (871) 7110784

Correo electrónico:mrg12208@uadec.edu.mx 